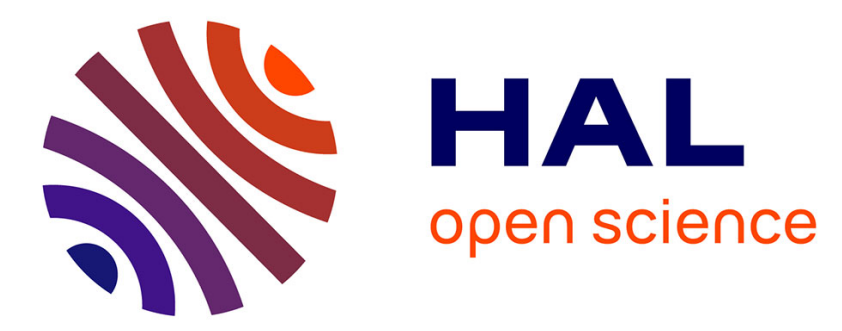

\title{
Études consulaires, études méditerranéennes. Éclairages croisés pour la compréhension du monde méditerranéen et de l'institution consulaire à l'époque moderne
}

Silvia Marzagalli

\section{- To cite this version:}

Silvia Marzagalli. Études consulaires, études méditerranéennes. Éclairages croisés pour la compréhension du monde méditerranéen et de l'institution consulaire à l'époque moderne. Cahiers de la Méditerranée, 2016, 93. hal-02171036

\section{HAL Id: hal-02171036 \\ https://hal.science/hal-02171036}

Submitted on 2 Jul 2019

HAL is a multi-disciplinary open access archive for the deposit and dissemination of scientific research documents, whether they are published or not. The documents may come from teaching and research institutions in France or abroad, or from public or private research centers.
L'archive ouverte pluridisciplinaire HAL, est destinée au dépôt et à la diffusion de documents scientifiques de niveau recherche, publiés ou non, émanant des établissements d'enseignement et de recherche français ou étrangers, des laboratoires publics ou privés. 


\section{Cahiers de la Méditerranée}

93 | 2016

Les consuls dans tous leurs états : essais et bibliographie (avant 1914)

\section{Études consulaires, études méditerranéennes. Éclairages croisés pour la compréhension du monde méditerranéen et de l'institution consulaire à l'époque moderne}

Silvia Marzagalli

\section{OpenEdition \\ Journals}

\section{Édition électronique}

URL : http://journals.openedition.org/cdlm/8469

ISSN : 1773-0201

Éditeur

Centre de la Méditerranée moderne et contemporaine

\section{Édition imprimée}

Date de publication : 15 décembre 2016

Pagination : 11-23

ISSN : 0395-9317

Ce document vous est offert par Université Nice Sophia-Antipolis

Référence électronique

Silvia Marzagalli, «Études consulaires, études méditerranéennes. Éclairages croisés pour la compréhension du monde méditerranéen et de l'institution consulaire à l'époque moderne », Cahiers de la Méditerranée [En ligne], 93 | 2016, mis en ligne le 15 juin 2017, consulté le 02 juillet 2019. URL : http://journals.openedition.org/cdlm/8469

Ce document a été généré automatiquement le 2 juillet 2019.

(c) Tous droits réservés 


\title{
Études consulaires, études méditerranéennes. Éclairages
} croisés pour la compréhension du monde méditerranéen et de l'institution consulaire à l'époque moderne

\author{
Silvia Marzagalli
}

1 Les historiens du monde méditerranéen de l'époque moderne ont depuis longtemps mis à profit les sources consulaires pour étayer leurs propos. Par sources consulaires, il faut entendre avant tout les dépêches envoyées par les consuls à leurs autorités de tutelle. Cette documentation est aussi la mieux préservée et la plus accessible, dans la mesure où elle était collectée et classée par les diverses administrations de l'État dont le consul représentait les intérêts. Certains ont également exploité les autres sources produites dans les consulats ou leur chancellerie, et rapatriées par la suite par l'État mandataire. Il s'agit aussi bien de registres d'état civil, que de procurations et autres actes de type notarié, voire de séries relatives à la navigation ou aux transactions économiques diverses. Si le recours à ce type de documents n'est pas spécifique aux études qui placent l'espace méditerranéen au cœur des propos, il a néanmoins été particulièrement important dans cette aire, non seulement en raison de la plus forte densité consulaire qui la caractérise, mais parfois aussi par l'absence d'autres sources aussi riches sur les objets qui intéressaient les chercheurs, ou à cause des inévitables limites de leurs compétences linguistiques dans un espace très marqué par la pluralité en la matière.

2 La réflexion que je voudrais présenter ici ne vise nullement à dresser le riche bilan de ces recherches, ni à mettre en avant son apport pour la compréhension de la Méditerranée de l'époque moderne, et non seulement car il serait impossible d'effectuer une telle synthèse dans le cadre d'un article. Ma perspective est différente. Je m'intéresserai ici davantage à 
la production qui, depuis une quinzaine d'années, alimente un chantier de recherche foisonnant, celui des études consulaires. Les historiens qui s'inscrivent dans cette mouvance ne mettent pas à profit la source consulaire en tant qu'élément auxiliaire pour élucider une problématique déterminée, en exploitant le contenu de l'information factuelle qu'elle livre. Ce sont plutôt l'institution consulaire et les consuls eux-mêmes qui sont au cœur de l'analyse. Ces chercheurs utilisent bien évidemment, en partie, les mêmes corpus documentaires que les études mentionnées plus haut, à savoir les écrits produits par les consuls. Mais ceux-ci sont interrogés à la lumière de ce qu'ils nous disent sur le fonctionnement des institutions, formelles ou informelles, dans lesquelles les consuls s'inscrivent (étatiques, négociantes, "nationales", confessionnelles, ou autres). Ces historiens s'évertuent en somme de manière croissante à comprendre ce que signifie être consul à l'époque moderne.

3 En mettant à profit cette production historiographique et quelques sources consulaires inédites, cet article se propose de mettre en avant ce que le renouveau de ce champ historiographique peut nous dire sur la Méditerranée et les logiques de fonctionnement qui se déploient en son sein, tout en interrogeant les spécificités de la fonction consulaire dans cette aire géographique. Les pistes suggérées ici s'articulent autour de trois éléments: l'étendue du maillage consulaire, les logiques qui président aux choix du personnel consulaire, et les diverses allégeances des consuls. Sans aucune prétention à l'exhaustivité, il s'agit plutôt de proposer quelques éléments de réflexion à la croisée des études consulaires et des études méditerranéennes qui pourraient alimenter les efforts de recherche à venir.

\section{Le maillage consulaire en Méditerranée : une chronologie et une densité spécifiques?}

4 La fonction consulaire naît en Méditerranée, qu'on la fasse remonter au proxène grec - en reprenant la fonction sans le terme-, ou aux représentants des nations des villes italiennes à la fin du Moyen-Âge, lorsque le terme de consul commence à être employé pour les désigner'. Mais est-ce seulement le poids de l'histoire qui rend compte de la concentration de postes autour du bassin méditerranéen à la fin du XVIII ${ }^{\mathrm{e}}$ siècle, lorsque l'ensemble des États européens s'est doté d'un système consulaire? Celle-ci est particulièrement évidente dès qu'on regarde la carte de l'emplacement des consulats français ${ }^{2}$, mais elle interroge aussi lorsqu'on se penche sur les cas suédois, danois, ou américain ${ }^{3}$, États qui, à la fin du XVIII ${ }^{\mathrm{e}}$ siècle, concentrent plus d'un quart de leurs postes consulaires autour de la Méditerranée. Vu l'insistance avec laquelle les historiens mettent généralement en avant l'importance de l'Atlantique dans le commerce international de cette époque, ce pourcentage a de quoi surprendre. Il invite ainsi aussi bien à interroger à nouveaux frais la prétendue marginalisation de la Méditerranée dans des échanges en voie de mondialisation ${ }^{4}$, qu'à poser la question des spécificités du commerce en Méditerranée qui pourraient nécessiter une présence plus importante de consuls.

5 On peut avancer, en guise d'hypothèse, que la densité d'acteurs et de normes en Méditerranée rend l'intermédiation consulaire plus utile qu'ailleurs. Daniel Panzac a clairement montré le rôle fondamental des consulats européens dans la rédaction des contrats de nolis entre chrétiens, musulmans et Grecs orthodoxes, Arméniens ou juifs, propre du commerce levantin, sur lesquels repose une partie considérable des échanges 
des produits issus des mondes ottoman et barbaresque ${ }^{5}$. On ne reviendra pas sur le rôle diplomatique des consuls dans les régences, sur lequel l'historiographie a tout particulièrement insistét. Je suggérerai pour ma part l'hypothèse additionnelle que le monde méditerranéen, avec sa forte urbanisation et ses productions complémentaires entre ses rivages - qu'on pense au commerce traditionnel de céréales et d'huile d'olive, ou aux nouveaux courants de trafic engendrés au XVIII ${ }^{e}$ siècle par l'essor des productions antillaises $^{7}$ - ait attiré plus que d'autres espaces maritimes les transporteurs étrangers, sans qu'il y ait toujours eu pour autant de véritables communautés marchandes sur place. C'est le cas pour les Scandinaves, mais cela semble aussi être, d'après mes recherches en cours, celui des Américains. Pour ces transporteurs, l'éventuelle absence d'une communauté marchande établie capable de fournir aux capitaines des informations précises sur les us et coutumes de la place, mais aussi sur ceux en vigueur dans d'autres ports méditerranéens, tout en connaissant parfaitement la nature des produits qui répondent au mieux aux besoins des pays d'où les navires sont originaires, a pu rendre les bons offices des consuls d'autant plus nécessaires.

6 L'ouvrage qui paraîtra prochainement sur la fonction marchande des consuls apportera une riche moisson de données ${ }^{8}$. Il permettra de mieux cerner la multiplicité des apports et des interventions de ces derniers dans le bon déroulement des transactions marchandes, et de dégager les éventuelles spécificités de l'aire méditerranéenne. Il faudra toutefois aussi veiller à saisir précisément de quels intérêts marchands il est question à chaque fois, car il est inenvisageable de les concevoir comme un ensemble cohérent et homogène9. Il se peut d'ailleurs que la multiplicité d'acteurs issus de pays, d'institutions et de différents groupes d'intérêt en Méditerranée à l'époque moderne, sans que l'un d'entre eux parvienne à exercer une véritable hégémonie ${ }^{10}$, constitue un élément ultérieur pouvant rendre compte de la densité consulaire particulière dans cette aire géographique. Les consuls représenteraient dès lors les intérêts marchands qui réussissent à obtenir l'oreille des hommes au pouvoir dans l'État qui les désigne. Le cas suédois conforte cette hypothèse : Leos Müller montre que le système consulaire suédois est avant tout au service du négoce de Stockholm, qui s'affirme parfois contre les intérêts des négociants des autres villes du royaume ${ }^{11}$. Le constat de Katerina Galani à propos du maillage consulaire anglais en Méditerranée vers la fin du XviII siècle semble aller dans le même sens. En étudiant 1569 lettres écrites dans le cadre de sa fonction par l'ambassadeur à Constantinople, Sir Robert Ainslie (1775-1794), dont une partie considérable est adressée aux consuls britanniques, elle montre comment il est impossible de séparer les intérêts de la Compagnie du Levant des enjeux diplomatiques de la couronne, qui se doublent en effet d'enjeux commerciaux ${ }^{12}$. Si cette conclusion apporte de l'eau au moulin de ceux qui prônent une prise en compte systématique des consuls dans l'étude des relations internationales ${ }^{13}$, elle conforte aussi l'idée que le consul ait été le représentant de groupes d'intérêts établis, éventuellement en concurrence avec d'autres.

7 Les coalitions qui se forment parfois pour demander le renvoi d'un consul - que ce soit celui du consul hollandais Van Dam à Smyrne dans la seconde moitié du XVII ${ }^{\mathrm{e}}$ siècle, du Français Laurent Soleil à Barcelone au début du XVIII ${ }^{\mathrm{e}}$ siècle, ou du consul des États-Unis Jean Mathieu à Naples en $1803^{14}$ - dévoilent ainsi les tensions d'ordre commercial sousjacentes, même si les arguments mis en avant par les pétitionnaires font appel à d'autres ressorts, davantage susceptibles de convaincre leurs destinataires, tels que corruption, incompétence, inefficacité, manque d'intérêt pour les affaires de la nation. Autrement dit, 
les conflits entre consul et communauté «nationale» ou capitaines, qui jalonnent la littérature et percent même à travers les sources consulaires à condition que le consul juge opportun de les mentionner dans sa correspondance, pourraient révéler aussi d'autres dynamiques que la seule résistance à l'emprise de l'État, et pointer plutôt vers des enjeux de pouvoir et de contrôle des marchés entre groupes concurrents. Si l'étude des procédures de nomination, et notamment des recommandations et candidatures spontanées, pourrait s'avérer un champ fécond de recherche pour tracer les réseaux qui se mobilisent à cette occasion ${ }^{15}$, la prise en compte des intérêts propres des consuls ajoute une couche de complexité de plus dans la compréhension des dynamiques à l'œuvre autour de l'établissement des consulats et de leur gestion.

\section{Le personnel consulaire, entre désignation et autopromotion}

Les études entreprises sur le personnel consulaire ont jeté les bases de nouveaux questionnements. Tout d'abord, il est désormais acquis que la fonction consulaire sous l'Ancien Régime obéit à une logique d'autoreproduction, dans laquelle fils et beau-fils succèdent souvent aux pères, et les neveux aux oncles. Cette logique n'est évidemment pas spécifique à la fonction consulaire, dans la mesure où elle se retrouve dans bien d'autres corps d'État, et que la reproduction socio-professionnelle au sein de la famille est par ailleurs la norme à l'époque moderne. Il est néanmoins significatif que, dans le cas français, le passage du consul, sous Colbert, du statut d'office vénal héréditaire à celui d'une commission révocable n'empêche en rien la formation de dynasties consulaires. Les études de Anne Mézin pour la France et de Jesús Pradells Nadal pour l'Espagne des Bourbons permettent de puiser de nombreux exemples ${ }^{16}$, qui semblent conforter l'idée que pour les États il s'agit avant tout de disposer d'un personnel offrant quelques garanties de compétences, et révocable en cas de nécessité. À une époque où aucune formation spécifique n'est dispensée au personnel consulaire - en dehors du cas particulier de l'école de langues pour les drogmans -, les fils apprennent le métier sur le tas, en héritant aussi du réseau relationnel du père. Cet héritage facilite la maîtrise des circuits qui leur permettent de se procurer les informations et d'apprendre les filtres qu'il convient de leur appliquer avant de les transmettre aux supérieurs ${ }^{17}$.

Le phénomène se retrouve aussi pour les pays qui s'appuient sur des consuls-négociants, à l'instar de la Suède, où la famille Butini-Fölsch exerce pendant cinq générations, de 1734 à 1881, la fonction de consul à Marseille ${ }^{18}$. La correspondance de François Philippe Fölsch, en cours de publication, permet de montrer comment les consuls poursuivent de manière systématique l'effort d'autopromotion des intérêts lignagers, mais aussi la relative disponibilité de la part des autorités de tutelle à consentir à la satisfaction des demandes jugées légitimes ${ }^{19}$. En insistant à onze reprises au moins auprès de ses supérieurs, François Philippe Fölsch parvient ainsi dans les années 1780 à se faire nommer consul général de Provence, alors qu'il était jusque-là seulement consul de Suède à Marseille ${ }^{20}$. Jean Vierne obtient, quant à lui, sans difficulté en 1771, après les avoir sollicitées, ses patentes de consul de Prusse à Nice d'abord, de l'ensemble des ports du royaume sarde ensuite (c'est-à-dire aussi de Villefranche et Oneglia), alors que sa demande présentée en 1786 visant à percevoir un salaire est refusée sans ambages ${ }^{21}$. 
Si ce type de fonctionnement, fondé sur une relation de protection auprès d'un « patron » au ministère, connu directement ou par un proche dans une mesure qui resterait à préciser, est propre à l'Ancien Régime et ne présente pas un caractère "méditerranéen " spécifique, du moins pas au XVIII ${ }^{e}$ siècle, il serait à vérifier dans quelle mesure le poids des dynasties consulaires est plus important ici qu'ailleurs, ce qui pourrait renvoyer à une plus forte agency de la part des consuls méditerranéens dans leur relation avec les autorités de tutelle et aux particularités du commerce dans la mer Intérieure (ou dans une partie de celle-ci).

Plus généralement, la volonté de léguer le poste consulaire à un enfant, l'appel au patronage des supérieurs, les candidatures spontanées, accompagnées souvent de recommandations expressément sollicitées, posent la question de l'intérêt d'être consul à l'époque moderne ${ }^{22}$. L'intérêt pécuniaire, évident dans le cas des consuls salariés, ne peut jamais être exclu. Il serait utile, à cet égard, d'étudier de manière systématique les formes de rétribution et les défraiements que les États sont prêts à consentir, qui varient par ailleurs dans le temps et dans l'espace pour un même État, introduisant des différences de statut entre les consuls de la même place. Les exemples suivants, issus de trois consulats suédois en Méditerranée, montrent que les consuls jouent sur ces différences pour plaider leur cause et essayer d'obtenir une pension annuelle, tout en rappelant leur inscription dans le cadre d'une relation de patronage.

Dès 1782, alors qu'il n'a hérité de son poste que depuis deux ans, François Philippe Fölsch signale que

[1]a cour du Danemark qui avait donné à son consul ici le consulat général de Provence, Sète et tout le Languedoc avec de très bons droits de consulat, ayant égard aux dépenses de sa place et à ses services ainsi qu'à la manière dont il s'est comporté (comme moi) à l'occasion des ambassadeurs que l'empereur du Maroc nous avait adressés, vient de lui accorder une pension d'environ 1500 livres.

Deux ans plus tard, il écrit à son supérieur que «le roi d'Espagne vient de gratifier son consul général en cette ville de la croix de l'ordre de Charles III et d'une pension de mille livres $»^{23}$, puis, en 1785 , il demande ouvertement au comte Creutz une gratification sous forme d'un remboursement forfaitaire de ses frais :

Et avec la plus respectueuse confiance dans les bontés et la justice de Votre Excellence je prends la liberté de lui observer que par la situation et l'importance de cette ville qui la rend le principal port de la Méditerranée, je suis obligé à une correspondance exactement suivie avec plusieurs des collèges royaux, presque tous les consuls de Sa Majesté et nombre d'autres personnes, les relations que le grand abord de cette place m'attire se multiplient à l'infini et m'occasionnent des ports de lettres considérables, dont je n'ai pas le moindre remboursement étant sans appointements. Votre Excellence connaît les divers traitements que Sa Majesté fait à plusieurs de ses consuls dont quelques-uns résident dans des ports qui exigent moins de frais de représentation et moins de ports de lettres, par exemple, si je suis bien informé, le consul à Sète a 200 ou 250 rixdaler specie des bienfaits du roi, il y a cependant une bien grande différence et des frais et des ports de lettres et même des occupations de sa place à celle-ci. Je supplierai donc très humblement Votre Excellence de prendre cet objet en considération et de daigner me faire obtenir des bontés de Sa Majesté un petit traitement annuel relatif à ce que je viens d'avoir l'honneur d'exposer. En cela Votre Excellence me donnerait une preuve qui m'est bien nécessaire, de la gracieuse protection dont elle veut bien m'honorer ${ }^{24}$.

14 En 1794, François Vierne, consul de Suède à Nice, sollicite une rémunération en comparant sa situation à celle des autres consuls de sa place : 
J'ai l'honneur de représenter à Votre Excellence que depuis dix à douze années que je suis revêtu de l'honorable charge de consul de Sa Majesté le roi de Suède, je n'ai joui d'aucune pension, ni même la permission de percevoir le droit de consulat sur les vaisseaux suédois qui abordent dans mon département, ce qui m'aurait couvert des dépenses attachées audit consulat, car tous les consuls des nations étrangères dans ce port la majeure partie sont pensionnés et reçoivent le consulat sur les vaisseaux de leurs nations. Je viens donc supplier Votre Excellence de s'intéresser en ma faveur auprès de Sa Majesté afin que je puisse obtenir une petite pension de laquelle j'aurai une reconnaissance éternelle à Votre Excellence ${ }^{25}$ des droits consulaires. Leurs missives sont à cet égard explicites. En refusant en 1800 le poste de consul des États-Unis à Rome, que Giovanni Battista Sartori avait quitté en 1800, John S. Cogdell écrit en 1806 au secrétaire d'État Madison :

Comme aucun salaire n'est attaché à cette fonction et qu'il n'y a aucune probabilité, vu la situation de Rome, que les demandes de certificats consulaires soient fréquentes, les perspectives mêmes de me soutenir seraient désespérées ${ }^{27}$.

En d'autres termes, les consuls ne prennent leurs fonctions que si le poste correspond à leurs attentes : informé en mars 1802 de sa nomination en tant que consul des États-Unis à Sète, James Anderson met trois ans avant de rejoindre son poste, qu'il quitte moins de trois mois plus tard:

[...] en raison de la conviction profonde de l'impossibilité de faire face aux dépenses en résidant à Sète, j'ai pris la décision de m'établir à Marseille, où j'espère avoir plus de succès ${ }^{28}$.

18 Les demandes de promotion à des postes plus lucratifs témoignent elles aussi d'une stratégie bien comprise d'utilisation des ressources qu'offre la fonction consulaire pour commencer une carrière ailleurs. Ces stratégies sont complémentaires à celles des dynasties bien ancrées dans une place et qui préfèrent œuvrer pour conserver le poste consulaire au sein de la famille et le transmettre d'une génération à l'autre.

19 Il est enfin indéniable, même si cela demeure impossible à quantifier, que les individus s'attendent à des avantages divers attachés à leur fonction consulaire, en termes de surface positionnelle et de protection face aux vicissitudes politiques par exemple. En juillet 1803, quelques mois à peine après la rupture de la paix d'Amiens, le consul des États-Unis à Gênes, Wollaston, explique à sa tutelle que sa maison de commerce a subi des revers l'amenant à déposer le bilan, et il exprime le souhait de garder son poste en dépit de l'absence d'émoluments significatifs liés à sa fonction (un seul navire américain était arrivé dans le port dans les six mois précédents). Il déclare à ce propos que le poste équivaut à une grande recommandation commerciale au moment où la reprise de la guerre et la neutralité américaine lui laissent envisager de se remettre en affaires ${ }^{29}$. En sachant que Wollaston est un sujet britannique, la protection consulaire peut lui donner 
des avantages non négligeables dans une ville sous contrôle français, faute d'avoir réussi à obtenir la nationalité américaine ${ }^{30}$. Son homologue à Trieste, William Riggin, réussit à éviter la contribution extraordinaire imposée aux négociants de la ville au lendemain de son occupation par les armées napoléoniennes en novembre 1806, en faisant valoir sa qualité de consul d'une nation en paix avec l'Empire français ${ }^{31}$.

Les répercussions réelles d'une nomination au consulat en termes d'accroissement du chiffre d'affaires mériteraient également d'être élucidées à partir de quelques études de cas, là où les sources le permettent. En l'absence de contacts préétablis avec une place marchande, un consul-négociant peut raisonnablement espérer que les capitaines s'adressent à lui non seulement pour leurs démarches administratives, mais aussi pour vendre leur cargaison et acheter celle de retour. À Palerme, le consul des États-Unis, qui est au demeurant un négociant britannique, est le consignataire de deux tiers de la centaine de navires américains entrés dans le port en 1806 et $1807^{32}$.

21 Si l'obtention d'un consulat est perçue comme une marque de reconnaissance, les consuls veillent aussi à prévenir tout événement qui pourrait engendrer des soupçons quant à l'appui inconditionnel de leur tutelle et entacher ainsi leur réputation. Thomas Appleton, consul des États-Unis à Livourne, tente par exemple à plusieurs reprises d'obtenir la charge d'agent naval, c'est-à-dire la faculté d'approvisionner les unités de la Marine américaine lorsqu'elles se trouvent dans son port. Dans un post-scriptum à sa lettre du 15 janvier 1802, il remarque qu'il est le seul consul parmi les dix-huit de la place à ne pas avoir cette fonction, et le seul consul américain en Méditerranée à avoir éprouvé une telle " disgrâce " $33^{3}$. Même si c'est l'honneur qui est mis en avant, la fonction entraîne aussi des commissions :

Je vous prie de croire que les émoluments pécuniaires qui peuvent en dériver ne constituent qu'une partie infime dans ma requête; mais comme il a toujours été considéré dans ce port comme une fonction inséparable des devoirs d'un consul, une modification dans les us et coutumes de la place entrainerait nécessairement des conséquences ${ }^{34}$.

Son confrère à La Valette, le Maltais Joseph Pulis, est encore plus direct: il conteste ouvertement le choix d'un négociant britannique comme agent naval des États-Unis à Malte, estimant que le choix du commodore américain est " très injuste », car il amène le public à croire que le consul n'est pas en état de rendre les services inhérents à sa fonction ${ }^{35}$.

En changeant de focale et en déplaçant le regard des systèmes consulaires aux consuls, la question de l'agency de ces derniers est ainsi posée avec force. D'autres éléments semblent inviter à creuser dans cette direction.

\section{Quelles allégeances?}

Les sources et les études consulaires existantes permettent en effet de constater, en croisant les données, que certains consuls exercent des fonctions consulaires pour plusieurs États à la fois, ce qui constitue une entrée relativement simple pour poser plus largement la question complexe de leurs allégeances. On se limitera à quelques exemples piochés au hasard des lectures. Henrick Gottfried Brandel, consul de Suède à Alger de 1766 à 1792, y est aussi consul d'Autriche depuis $1784^{36}$. Pierre-Jean de Bertellet, consul de France à Livourne de 1731 à 1763 , y devient également consul de Suède en $1745^{37}$. Le premier consul des États-Unis à Gibraltar, James Simpson, est aussi consul de Russie ${ }^{38}$. 
Plus original, Giovanni Battista (alias John Baptist) Sartori, premier consul des États-Unis à Rome, quitte son poste dès 1800 en confiant le consulat à son père, et émigre à Trenton, au New Jersey, où il essaie de se faire accréditer comme consul des États pontificaux aux États-Unis ${ }^{39}$.

Ce n'est que progressivement, comme l'a souligné Marcella Aglietti, que l'obligation d'être de la nationalité du pays qu'on représente et de limiter les allégeances à un seul État se précise ${ }^{40}$. Ainsi, en 1798, le consul de Raguse (Dubrovnik) à Marseille, Nicolo' Pagano, donne sa démission car la nouvelle constitution de la République de Gênes - nous sommes dans le Triennio révolutionnaire - interdit à tout ressortissant de servir une puissance étrangère, alors que ce consul est Génois ("Sono io naturale di quella capitale, vi ho Parenti e Beni $»)^{41}$. La règle, toutefois, comprend longtemps bien des exceptions ${ }^{42}$, qui sont parfois si nombreuses qu'elles viennent à constituer la norme. En dépit d'une législation qui prévoit depuis 1784 que les consuls des États-Unis soient des citoyens américains ${ }^{43}$, les premiers consuls nommés autour du bassin méditerranéen par la jeune république sont majoritairement des étrangers : sur les seize premiers titulaires nommés aux postes créés par les États-Unis en Méditerranée occidentale avant 1815, six seulement sont des citoyens américains. Parmi les quinze remplaçants ou successeurs désignés sur ces mêmes postes avant 1815, onze sont Américains. On aurait vite fait de conclure à une rapide évolution visant à respecter davantage la lettre de la loi. Or, la réalité est plus complexe. Tout d'abord, car l'évolution n'est pas linéaire. Ainsi, si Joseph Barnes, nommé consul en Sicile en 1802, est un ressortissant des États-Unis - fort absentéiste au demeurant, ce qui lui coûte son poste-, les deux consuls qui le remplacent en 1805, à Palerme et à Messine, sont des négociants britanniques que Barnes avait accusés d'incompétence, voire de malversations dans plusieurs lettres, alors qu'il essayait d'obtenir le consulat général pour l'ensemble des ports du royaume de Naples et de Sicile, en obtenant aussi, par la même occasion, le limogeage du Français Mathieu, consul des États-Unis à Naples ${ }^{44}$. Force est de constater aussi que le taux de refus ou de non-prise de service est nettement plus élevé parmi les Américains que parmi les étrangers. Le phénomène est particulièrement évident pour les primo-nommés - deux consuls américains sur six seulement prennent effectivement fonction, contre neuf sur dix parmi les étrangers -, mais il s'observe aussi par la suite, avec quatre des onze consuls américains nommés qui ne rejoignent pas leur poste, alors que tous les étrangers entrent en fonction. Ces données confirment que les gouvernements doivent composer avec la réalité du terrain. Il est possible que la nature du commerce méditerranéen ne leur offre parfois d'autre recours que celui de s'appuyer sur des ressortissants d'autres pays déjà sur place, et ce d'autant plus que tout poste consulaire ne présente pas la même attractivité, et que les avantages ne sont pas nécessairement partout très intéressants. En bref, il se pourrait qu'une compréhension plus fine de la composition du personnel consulaire requière de renverser la perspective et de partir de l'agency des individus œuvrant pour être reconnus comme consuls, plutôt que de l'État, conçu comme impersonnel, qui choisirait librement les consuls à nommer.

Cette approche soulève toute une série d'interrogations qui méritent des recherches spécifiques. Tout d'abord, il faudrait répertorier de manière plus systématique les consuls en poste à un moment donné pour identifier la part de ceux qui servent plusieurs États à la fois, et étudier leur comportement en cas d'éventuel conflit entre ces puissances. En parallèle, il conviendrait de repérer également les États dont ils sont les sujets, ainsi que ceux de leur réseau de parenté, car il est possible que certains groupes "nationaux » 
aient réussi à pénétrer en profondeur les services consulaires en Méditerranée à certains moments ou dans certains lieux, en reflétant au passage les logiques propres au commerce en Méditerranée, car c'est sur les négociants que s'appuie avant tout le choix des consuls, indépendamment de leurs allégeances «nationales». Force est aussi de constater que les alliances matrimoniales de ces consuls ne se font pas uniquement avec des ressortissantes de leur pays, loin de là. Le phénomène n'est par ailleurs pas surprenant, si l'on considère que la plupart des systèmes consulaires de l'époque moderne reposent sur des négociants au profil hautement internationalisé. Plus largement, il s'agirait de repérer les différents réseaux dans lesquels s'inscrivent les consuls en Méditerranée et au sein desquels se déploie leur action. Pour la plupart d'entre eux, les correspondances consulaires se doublent de celles à caractère négociant, et concourent à en enrichir le contenu. François Philippe Fölsch est pleinement conscient de la valeur ajoutée que ses divers réseaux d'appartenance apportent à son information consulaire, et il ne manque pas de la mettre en avant :

Mes relations particulières, ma correspondance assez multipliée me mettent dans le cas pour l'avantage et l'accroissement du commerce suédois [...], de devoir être instruit de tout ce qui peut directement ou indirectement, concourir à faire fructifier mon service, objet unique et cher à mon ambition ${ }^{45}$.

Au-delà de la fonction consulaire, de l'appartenance plus ou moins marquante à une communauté d'origine "nationale » ou confessionnelle, et au milieu socio-professionnel du négoce pour la plupart d'entre eux, d'autres identités relient les consuls entre eux et à d'autres individus, élargissant ainsi le cercle des contacts et des relations potentiellement mobilisables, mis au service les unes des autres. Au siècle des Lumières, en particulier, bon nombre de consuls participent aux réseaux de sociabilité maçonnique, dont PierreYves Beaurepaire a rappelé qu'ils s'inscrivent à leur tour dans des enjeux de pouvoir qui dépassent au demeurant les horizons méditerranéens ${ }^{46}$. Le choix des individus avec qui les consuls interagissent est par ailleurs déterminé par le contexte spécifique à leur poste, notamment en terre d'Islam: Christian Windler apporte de nombreux exemples de liens entre familles consulaires à Tunis aux allégeances confessionnelles multiples, y compris entre consuls appartenant à des États en guerre entre eux. Il y voit la confirmation de « la portée des réseaux qui échappaient aux rationalités nationales ${ }^{47}$.

Arrivées aujourd'hui à une certaine maturité, les études consulaires enrichissent désormais nos questionnements sur le fonctionnement du monde méditerranéen. Au-delà des apports réels dans les champs de l'histoire diplomatique, économique et institutionnelle, elles peuvent aider à reformuler nos interrogations à partir de la prise en compte des comportements et des stratégies des acteurs qui, par la toile de leurs relations et en poursuivant les objectifs individuels, dynastiques et collectifs qui leur sont propres, contribuent à configurer le système et à le faire évoluer.

De son côté, la connaissance des mécanismes propres au monde méditerranéen, notamment en matière d'échanges et de navigation, et plus largement de pratiques de l'interrelation, semble ouvrir quelques pistes susceptibles d'éclairer le fonctionnement de l'institution consulaire et ses spécificités méditerranéennes. Le berceau méditerranéen dans lequel les consuls ont vu le jour n'a pas fini de nourrir la recherche. 


\section{NOTES}

1. Voir Jörg Ulbert, «Introduction », dans Jörg Ulbert et Gérard Le Bouëdec (dir.), La fonction consulaire à l'époque moderne, Rennes, Presses universitaires de Rennes, 2006, p. 9-20.

2. Anne Mézin, Les consuls de France au siècle des Lumières (1715-1792), Paris, Ministère des Affaires étrangères, Direction des archives et de la documentation, 1997.

3. En 1800 , on dénombre 21 ports méditerranéens parmi les 79 postes consulaires suédois dans le monde : Leos Müller, Consuls, Corsairs, and Commerce. The Swedish Consular Service and Long-distance Shipping, 1720-1815, Uppsala, Uppsala University, 2004, p.42-43. Le Danemark a 15 postes consulaires en Méditerranée sur 40 ouverts en Europe et dans l'espace méditerranéen en 1787 : Pierrick Pourchasse, «Les consulats, un service essentiel pour le monde négociant : une approche comparative entre la France et la Scandinavie », dans Jörg Ulbert et Gérard Le Bouëdec (dir.), La fonction consulaire..., op. cit., p. 199. En 1800, les États-Unis ont 16 postes en Méditerranée sur 57 ouverts dans le monde : Debra J. Allen, Historical Dictionary of US Diplomacy from the Revolution to Secession, Lanham, Scarecrow Press, 2012, appendix D.

4. Arnaud Bartolomei et Silvia Marzagalli (dir.), «La Méditerranée dans les circulations atlantiques au XVIII ${ }^{\mathrm{e}}$ siècle ", dossier thématique, Revue d'histoire maritime, $\mathrm{n}^{\circ}$ 13, 2011.

5. Daniel Panzac, La caravane maritime: marins européens et marchands ottomans en Méditerranée (1680-1830), Paris, CNRS éditions, 2004.

6. Christian Windler, La diplomatie comme expérience de l'Autre. Consuls français au Maghreb (1700-1840), Genève, Droz, 2002.

7. Pour deux produits méditerranéens majeurs, voir Jean-Louis Miège (dir.), Les céréales en Méditerranée. Histoire, anthropologie, économie, Paris, CNRS, 1993 et L'huile d'olive en Méditerranée: histoire, anthropologie, économie de l'Antiquité à nos jours, Aix-en-Provence, Institut de recherches et d'études sur le monde arabe et musulman, 2013. Sur le virage antillais de Marseille, voir Charles Carrière, Négociants marseillais au XVIII siècle, Marseille, Institut historique de Provence, 1973, 2 vol.

8. Arnaud Bartolomei, Guillaume Calafat, Mathieu Grenet et Jörg Ulbert (dir.), De l'utilité commerciale des consuls. Consuls et réseaux consulaires en Europe et dans le monde méditerranéen (XVII $X I X^{e}$ siècles), Madrid, Casa de Velázquez, à paraître.

9. Voir, sur ce point, les remarques de Mathieu Grenet, «Consuls et "nations" étrangères : état des lieux et perspectives de recherche ", dans ce même dossier des Cahiers de la Méditerranée. Vont dans ce sens aussi les conclusions de Arnaud Bartolomei, «De la utilidad comercial de los cónsules. Problemáticas y estado de la cuestión (Europa y el mundo mediterráneo, siglos XVII, XVIII y XIX)», dans Marcella Aglietti, Manuel Herrero Sánchez et Francisco Zamora Rodríguez (dir.), Los cónsules de extranjeros en la Edad Moderna y a principios de la Edad Contemporánea , Madrid, Doce Calles, 2013, p. 246-258.

10. Pour l'absence d'hégémonie politique dans la Méditerranée moderne, voir Molly Greene, "Beyond the Northern Invasion: The Mediterranean in the Seventeenth Century», Past \& Present : A Journal of Historical Studies, $\mathrm{n}^{\circ} 174$, février 2002, p. 42-71.

11. Leos Müller, Consuls..., op. cit.

12. Katerina Galani, British Shipping and Trade in the Mediterranean in the Age of War, 1770-1815, PhD thesis, University of Oxford, 2010, p. 38-42.

13. Voir le plaidoyer en ce sens de Ferry de Goey dans ce dossier des Cahiers de la Méditerranée ainsi que bien évidemment l'ouvrage fondamental de Christian Windler, La diplomatie..., op. cit., 
qui a attiré le premier l'attention sur les processus de négociation des normes par les consuls. Sur la relation problématique entre histoire consulaire et histoire diplomatique, voir aussi Halvard Leira et Iver B. Neumann, «The many past lives of the consul», dans Jan Melissen et Ana Mar Fernandez(dir.), Consular Affairs and Diplomacy, Leyde et Boston, Martinus Nijhoff, 2011, p. 225-246.

14. Thierry Allain, «L'information comme instrument de combat : le consulat de Jacob van Dam à Smyrne (1668-1688)», dans Silvia Marzagalli (dir.), Les consuls en Méditerranée, agents d'information, en collaboration avec Maria Ghazali et Christian Windler, Paris, Classiques Garnier, 2015, p. 81-97 ; Julien Sempéré, Le consulat français de Barcelone (1679-1716) : Guerre et commerce en Méditerranée, thèse de doctorat, Université de Paris 1 - Panthéon-Sorbonne, 2014, p. 405-414 ; National Archives and Record Administration (Washington), désormais NARA, RG59 Naples, lettre datée Naples, $1^{\mathrm{er}}$ mai 1803, adressée à James Madison et signée par six capitaines et subrécargues américains à Naples, qui dénoncent l'incompétence du consul des États-Unis, le Français Jean Mathieu, et l'accusent de négliger les intérêts de la nation ( who feels no attachement to the country he represents, and has not even prudence enough to conceal it »), en demandant qu'il soit remplacé par le Prussien Fredrick Degen, faute de citoyens américains établis sur place ( as there is no American citizen established in this place »). Le contenu de cette missive est confirmé par plusieurs négociants américains connus, qui déclarent avoir été pour affaires à Naples à des périodes différentes (ibid., lettre du $1^{\mathrm{er}}$ septembre 1803). Degen accuse réception de sa nomination au consulat de Naples le 17 décembre 1805. Après une faillite probablement frauduleuse, son successeur n'hésite pas à le dénoncer ("His want of integrity is the frequent subject of conversation, yet he still remains the patronage of our Country ", ibid., Naples, 22 décembre 1810, Alexander Hammet à Robert Smith.

15. Sans avoir eu le temps d'approfondir cet aspect, il est frappant de constater, à la lecture des correspondances consulaires américaines, le poids que la recommandation ou la connaissance personnelle des hommes au pouvoir semble jouer dans la nomination d'un consul. Une étude systématique mériterait d'être menée en mettant à profit la série Letters and recommendations consevée au NARA, dont je n'ai pas encore pu prendre connaissance.

16. Anne Mézin, Les consuls de France..., op. cit.; Jesús Pradells Nadal, Diplomacia y comercio. La expansión consular española en el siglo XVIII, Alicante, Universidad Instituto de Cultura Juan GilAlbert, 1992.

17. Pour un exemple du traitement des informations, de la collecte à la transmission aux autorités de tutelle, voir Pierre-Yves Beaurepaire et Silvia Marzagalli, «"Pour les nouvelles les plus fraîches et les plus certaines". La correspondance du consul suédois à Marseille à la fin du XVIII ${ }^{\mathrm{e}}$ siècle comme instrument d'information et d'autopromotion ", dans Silvia Marzagalli (dir.), Les consuls en Méditerranée..., op. cit., p. 219-238.

18. Johan Axel Almquist, Kommerskollegium och Riksens Ständers Manufakturkontor samt konsulsstaten 1651-1910 : administrativa och biografiska anteckningar, Stockholm, Norstedt, 1912-1915, p. 384.

19. Pierre-Yves Beaurepaire, Gustaf Fryksén, Silvia Marzagalli et Fredrik Thomasson (éd.), Un consul suédois en Méditerranée. La correspondance de François Philippe Fölsch, consul à Marseille (1780-1807), Paris, Classiques Garnier, 2 vol., à paraître.

20. Riksarkivet (Stockholm), Diplomatica Gallica 511, lettres du 14 et 18 décembre 1781, 14 et 18 janvier, $1^{\mathrm{er}}$ mars 1782 . Après avoir essuyé une première réponse nuancée, Fölsch réitère sa demande par ses lettres du 29 mars, $1^{\text {er }}, 12$ et 14 avril, et du 10 et 17 juin 1782.

21. Informations issues $d u$ Geheimes Staatsarchiv Preussischer Kulturbesitz, I. HA Rep. 9 Allgemeine Verwaltung, Z Lit. U, I. HA Rep. 9 Allgemeine Verwaltung, Z Lit. U, Fasz. 9, transmises par Jörg Ulbert, que je remercie ici sincèrement.

22. Ce thème fait l'objet d'une rencontre scientifique organisée par le Centre de la Méditerranée moderne et contemporaine à l'automne 2017. 
23. Riksarkivet (Stockholm), Diplomatica Gallica 511, lettres du $1^{\mathrm{er}}$ mars 1782 et du 6 août 1784 . Toutes les citations ont été modernisées pour ce qui concerne l'orthographe, l'usage des majuscules et la ponctuation. Les abréviations ont été explicitées en toutes lettres.

24. Ibid., $1^{\mathrm{er}}$ avril 1785.

25. Riksarkivet (Stockholm), Diplomatica Italica, Nizza, lettre du 11 avril 1794.

26. Riksarkivet (Stockholm), Diplomatica Italica, Genoua, lettre du 2 février 1793.

27. "There being no salary attach'd to this office and from the known situation of Rome no probability of frequent demands for consular certificates, the prospect of support even, wou'd be desperate ": NARA, RG59 Rome, John S. Cogdell à James Madison, Charleston (S.C.), 3 juin 1806.

28. "From a firm persuasion of the impossibility of paying my expenses by residing at Cette, I have taken the resolution of going to Marseille, where I hope to be more fortunate ", NARA, RG59 Cette, James Anderson à James Madison, 7 septembre 1805, accessible à l'URL http://founders.archives.gov/ documents/Madison/02-10-02-0283 (consulté le 5 août 2016).

29. NARA, RG59 Genoa, Frederick Hyde Wollaston à James Madison, 25 juillet 1803 : «I am the more desirous of retaining my Post in my present Circumstances as it being of little or no other emolument, it is, [a] very great mercantile introduction \& during the present War, the neutrality of the Un: States would enable me to extend a considerable branch of Trade here from thence, \& being well acquainted $w$ [ith] this Place \& its ressources by 18 years residence, having at the same time gained the good will of the Government \& Inhabitants, I deem myself better able to give general Satisfaction than a new comer ". Accessible à l'URL http://founders.archives.gov/documents/Madison/02-91-02-0786 (consulté le 5 août 2016).

30. Wollaston avait demandé sa naturalisation de manière réitérée : NARA, RG59 Genoa, lettres du 2 mars et 29 juin 1799, 27 juin 1800, 10 juin 1801, 30 janvier 1802.

31. NARA, RG59 Trieste, W. Riggin à James Madison, 6 janvier 1806.

32. NARA, RG84 Palermo, vol. 144.

33. "Only Consul of 18 in this place who is not charged with [the] Concerns of the Marine of their Nation "; " the alone Consul from the United States [in] the Mediterranean who has experienced this disgrace »: NARA, RG59 Leghorn, Thomas Appleton à James Madison (copie), 15 janvier 1802, accessible à l'URL http://founders.archives.gov/documents/Madison/02-02-02-0578 (consulté le 5 août 2016). 34. "I beg that you will believe, that the pecuniary emo[lu]ments which might Arise, have the smallest share in this my request; but as it has ever been Considered in this port, as a charge inseparable from the dut [ies] of a Consul, it will therefore have that tendency which a deviation from the Usage and Custom of the place will most necessarily produce ", NARA, RG59 Leghorn, Thomas Appleton à James Madison, 28 septembre 1801, accessible à l'URL http://founders.archives.gov/documents/ Madison/02-91-02-0164 (consulté le 5 août 2016).

35. "Without any grounded reason it is very unfair to employ a Stranger in that Capacity [...] such an Action induced still the public to believe, that the Consul was not capable of rendering such Services as that office requires ", NARA, RG59 Malta, Joseph Pulis à James Madison, 5 juillet 1804, accessible à l'URL http://founders.archives.gov/documents/Madison/02-07-02-0425 (consulté le 5 août 2016).

36. Johan Axel Almquist, Kommerskollegium..., op. cit., p. 474. Riksarkivet (Stockholm), Diplomatica Gallica 511, lettre de François Philippe Fölsch, 4 août 1786.

37. Voir Anne Mézin, Les consuls de France, op. cit. Johan Axel Almquist, Kommerskollegium..., op. cit ., p. 395 et 467. Pierre-Jean de Bertellet demeure consul de Suède et de France à Livourne jusqu'à sa mort, survenue en 1763. L'un de ses fils, Louis-Antoine, lui succède alors au consulat de France, alors qu'un autre de ses enfants, Jean-Pierre, est consul de France à Carthagène de 1757 à 1786.

38. Note à la lettre de James Simpson à George Washington, 30 avril 1793, accessible à l'URL http://founders.archives.gov/documents/Washington/05-12-02-0399 (consulté le 10 juillet 2016). 39. Fanny Morton Peck, "A Roman Consul of the Nineteenth Century », Records and Studies of the US Catholic Historical Society, vol. 13, 1919, p. 61-83 ; Sartori épouse aux États-Unis une Française, dont le père, qui avait des intérêts à Saint-Domingue, avait épousé une Espagnole. Leo Francis 
Stock, "The Papal Consuls of Philadelphia ", Records of the American Catholic Historical Society, vol. 55, 1944, p. 178-189.

40. Voir Marcella Aglietti, «Le gouvernement des informations. L'évolution du rapport entre État et institution consulaire au milieu du XVIII ${ }^{\mathrm{e}}$ siècle ", Cahiers de la Méditerranée, $\mathrm{n}^{\circ}$ 83, 2011, p. 297-307, et Marcella Aglietti, L'istituto consolare tra Sette e Ottocento. Funzioni istituzionali, profilo giuridico e percorsi professionali nella toscana granducale, Pise, ETS, 2012, en particulier le chapitre 7 « Da console della "nazione" a console dello Stato », p. 297-334.

41. Archives d'État de Dubrovnik, LXXVI Diplomata et Acta, Prep ${ }^{18} 76$ - 3115, passim, et notamment la lettre du 11 août 1798 d'où est tiré le passage cité («je suis naturel de cette capitale, j'y ai de la famille et des biens »).

42. Anne Mézin a démontré que ni les conditions d'âge, ni celles de religion ne sont respectées dans le cas des consuls français : Anne Mézin, Les consuls de France..., op. cit., p. 14-16.

43. La résolution du Congrès du 16 mars 1784 réserve explicitement toute charge consulaire et vice-consulaire aux citoyens des États-Unis : William Barnes et John Heath Morgan, The Foreign Service of the United States: Origins, Development, and Functions, Washington D.C., Government printing office, 1961, p.33-34. Les données qui suivent s'appuient sur la reconstitution nominative des titulaires des postes consulaires américains en Méditerranée. Elle a profité de quelques instruments récents répertoriant leurs noms : Debra J. Allen, Historical Dictionary..., op. cit., et le site http://www.eafsd.org/. Les informations qu'ils livrent requièrent toutefois des vérifications systématiques et de compléments, issus pour l'essentiel des sources produites par les consuls, conservées au NARA, RG59, que j'ai entièrement dépouillées pour les postes consulaires en Méditerranée jusqu'en 1815. Une partie de ces correspondances est accessible en ligne : http://founders.archives.gov/.

44. Après avoir essayé en 1796 d'obtenir le consulat des États-Unis à Londres, Joseph Barnes brigue le consulat général du royaume de Naples et de Sicile. Plusieurs de ses lettres à ce sujet sont conservées au NARA, RG59 Palermo. Pour un exemple des accusations contre le vice-consul de Messine, voir sa lettre du 20 décembre 1802 à Thomas Jefferson, ibid., accessible à l'URL http:// founders.archives.gov/documents/Jefferson/01-39-02-0172 (consulté le 5 août 2016).

45. Riksarkivet (Stockholm), Diplomatica Gallica 512, 15 octobre 1790.

46. Pierre-Yves Beaurepaire, «Le cosmopolitisme maçonnique dans les villes méditerranéennes au XVIII siècle ", Cahiers de la Méditerranée, $\mathrm{n}^{\mathrm{0}}$ 67, 2003, p. 33-50.

47. Christian Windler, La diplomatie..., op. cit., p. 146.

\section{RÉSUMÉS}

Depuis une quinzaine d'années, les études consulaires ont considérablement élargi notre conception $\mathrm{du}$ fonctionnement de cette institution, qui trouve ses racines dans le monde méditerranéen. Cet article propose une réflexion sur les apports de ce chantier d'études à notre compréhension du monde méditerranéen à l'époque moderne, tout en suggérant que nos connaissances sur la nature de celui-ci peuvent éclairer à leur tour certaines caractéristiques propres au fonctionnement des consulats en Méditerranée. Plus particulièrement, sont ici questionnées l'étendue du maillage consulaire, les logiques qui président aux choix du personnel consulaire, et les diverses allégeances des consuls. 
Over the past fifteen years, consular studies have considerably broadened our perception of this institution, which finds its origin in the Mediterranean world. This paper suggests that this research is likely to enrich our understanding of the early modern Mediterranean world, and that reversely our knowledge of the nature of this environment can help understand some characteristics of the ways consulates worked in the Mediterranean. In particular, this paper presents reflections on the density of consulates, the choice of consuls, and their multiple allegiances.

\section{INDEX}

Mots-clés : études consulaires, Méditerranée, époque moderne, consuls

Keywords : consular studies, Mediterranean, early modern times, consuls

\section{AUTEUR}

\section{SILVIA MARZAGALLI}

Silvia Marzagalli, professeur d'histoire moderne à l'Université Côte d'Azur et membre de l'Institut Universitaire de France, s'intéresse aux circulations (Atlas de la Révolution française. Un basculement mondial, 1770-1804, avec Pierre-Yves Beaurepaire, Paris, 2016) et aux réseaux marchands, tout particulièrement aux mécanismes d'adaptation mis en place en temps de guerre. Parallèlement à ses recherches sur la navigation américaine (Bordeaux et les États-Unis, 1776-1815 : politique et stratégies négociantes dans la genèse d'un réseau commercial, Genève, 2015, et étude en cours sur les États-Unis et la Méditerranée), elle étudie le rôle des consuls, notamment dans la transmission des informations (Les consuls en Méditerranée, agents d'information, $\mathrm{XVI}^{e}-\mathrm{XX}^{e}$ siècle, sous la dir. de Silvia Marzagalli, en collaboration avec Maria Ghazali et Christian Windler, Paris 2015). Silvia.Marzagalli@unice.fr 